Musées, Patrimoine et Culture scientifiques et techniques

$155 \mid 2014$

septembre-octobre 2014

\title{
Expérimentations autour d'un parcours enfant au sein de la Grande Galerie de l'Évolution
}

\author{
Fabienne Noé-Stosic
}

\section{OpenEdition Journals}

Édition électronique

URL : http://journals.openedition.org/ocim/1444

DOI : $10.4000 /$ ocim. 1444

ISSN : 2108-646X

\section{Éditeur}

OCIM

\section{Édition imprimée}

Date de publication : 1 septembre 2014

Pagination : 24-28

ISSN : 0994-1908

Référence électronique

Fabienne Noé-Stosic, «Expérimentations autour d'un parcours enfant au sein de la Grande Galerie de I'Évolution », La Lettre de I'OCIM [En ligne], 155 | 2014, mis en ligne le 01 septembre 2016, consulté le 19 avril 2019. URL : http://journals.openedition.org/ocim/1444 ; DOI : 10.4000/ocim.1444

Ce document a été généré automatiquement le 19 avril 2019.

Tous droits réservés 


\section{Expérimentations autour d'un parcours enfant au sein de la Grande Galerie de l'Évolution}

Fabienne Noé-Stosic

\section{L'accueil du jeune public au sein de la Grande Galerie de l'Évolution}

1 Depuis l'ouverture de la Grande Galerie de l'Évolution (GGE) au Muséum national d'Histoire naturelle en 1994, l'accueil du jeune public est pris en charge par l'équipe de l'action pédagogique et culturelle à travers une programmation d'ateliers et de visites dédiée aux enfants dès l'âge de 3 ans. 
Le cabinet de curiosités des enfants dans l'espace Kiki.

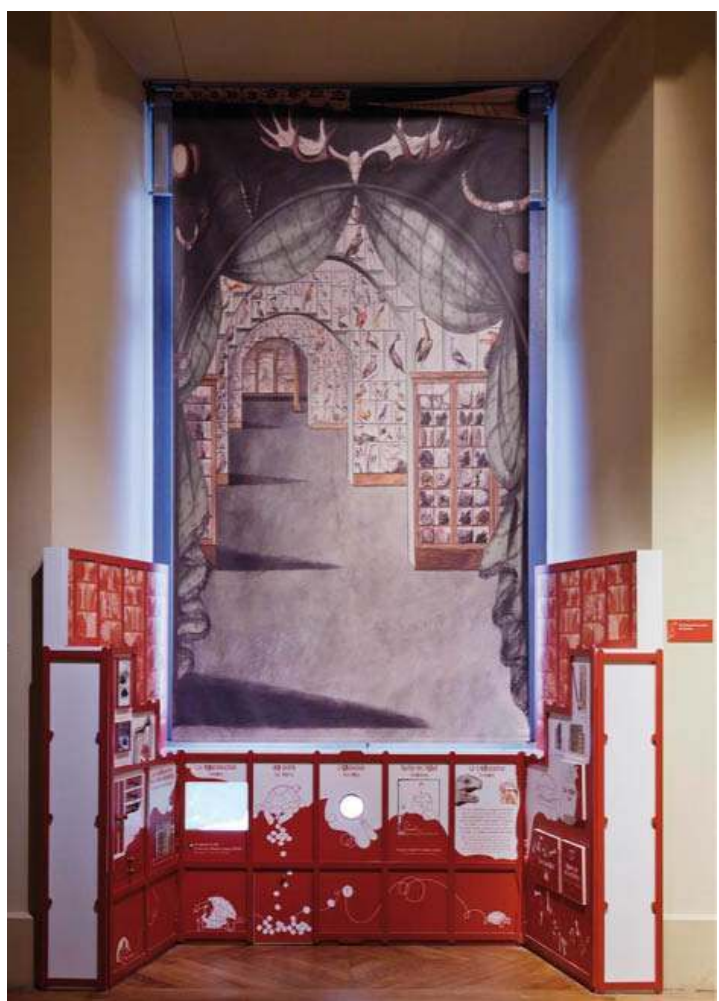

(c) MNHN/B. Faye

2 Ces ateliers ont été, pour la plupart, développés dans le cadre de partenariats avec des enseignants. Les thématiques sont donc en lien avec certaines notions de base de l'étude du vivant comme les régimes alimentaires, la locomotion, la découverte de la biodiversité des vertébrés, mais aussi en lien avec l'histoire des collections. Dans cette perspective, par exemple, l'animation "Le voyage de Théodore Floki" permet - à travers une saynète jouée par des animateurs - d'aborder les voyages naturalistes des XVIIIe et XIXe siècles et de répondre à la question des origines des collections exposées.

3 Dans les années 2000, la forte augmentation de la fréquentation des familles accompagnées d'enfants est une véritable opportunité pour mettre en place une programmation pour le public familial. La question est tout de même posée sur la nécessité de proposer à ce nouveau public une approche spécifique, et il est décidé que des ateliers conçus notamment avec l'aide d'artistes, de chorégraphes danseurs et de conteurs seront créés. Cette approche artistique et transversale entre l'art et les sciences donne ainsi une dimension moins formelle et scolaire à l'offre.

4 À partir de 2005, le projet de la Galerie des Enfants (GDE) se met en place, pour ouvrir ses portes en 2010. Il s'adresse spécifiquement à des enfants âgés de 6 à 12 ans accompagnés de leur famille. Les thématiques autour de la biodiversité, de l'impact de l'action de l'Homme sur son environnement et de son regard sur la préservation de la planète sont des points majeurs de cette Galerie.

5 C'est également à ce moment qu'une réflexion est suscitée par la direction du département des Galeries autour de la mise en place d'un parcours enfants au sein de la GGE qui pourrait s'adresser à des enfants de moins de 6 ans et qui complèterait ainsi 
l'offre de la GDE. C'est l'Année internationale de la Biodiversité en 2010 qui fournit l'occasion d'initier ce travail.

\section{Du parcours de la biodiversité à l'aménagement d'espaces pour les enfants}

6 En 2010, le Muséum prend part aux actions de l'Année internationale de la Biodiversité et l'équipe pédagogique du département des Galeries crée le "Parcours de la biodiversité" dans la Grande Galerie de l'Évolution. Les objectifs de ce parcours sont multiples et le propos s'articule autour de cinq questions : qu'est-ce que la biodiversité? Comment mesurer ses variations? Quelles en sont les causes? Quelles sont les conséquences de ces variations? Quelles actions éventuelles mener?

7 Il s'agit ici d'apporter des réponses aux questions en s'appuyant sur quelques animaux de la Grande Galerie insuffisamment valorisés et pourtant porteurs d'un véritable intérêt pédagogique.

8 Le parcours est composé d'un espace d'introduction qui permet de poser les cinq questions et de présenter le plan du parcours ponctué des différentes escales. Ces dernières sont matérialisées par la présence de bornes disposées dans les milieux marins et terrestres, en lien avec une thématique ou un animal choisi pour illustrer les menaces et les moyens mis en œuvre pour la protection de l'espèce.

Quatre animaux "phares" sont identifiés: le dodo, le narval, l'ours polaire et les ammonites. Le dodo fait son entrée dans ce parcours dans un espace qui doit attirer le public vers la salle des espèces menacées. Le narval est quant à lui intégré au parcours et devient le cœur du premier espace d'interprétation temporaire pour les jeunes enfants de la Grande Galerie. Situé en retrait dans un espace d'environ $100 \mathrm{~m} 2$ peu visité, il est l'occasion d'imaginer et de tester de nouveaux supports de diffusion.

En 2011, la tortue géante des Seychelles baptisée Kiki, animal emblématique de la ménagerie du Jardin des Plantes et naturalisée en 2010, fait son entrée à la GGE dans le prolongement de la salle des espèces menacées et disparues. Cet espace est pérenne contrairement aux deux autres précédemment évoqués. 
Le dispositif jeu pour enfant de l'espace Kiki.
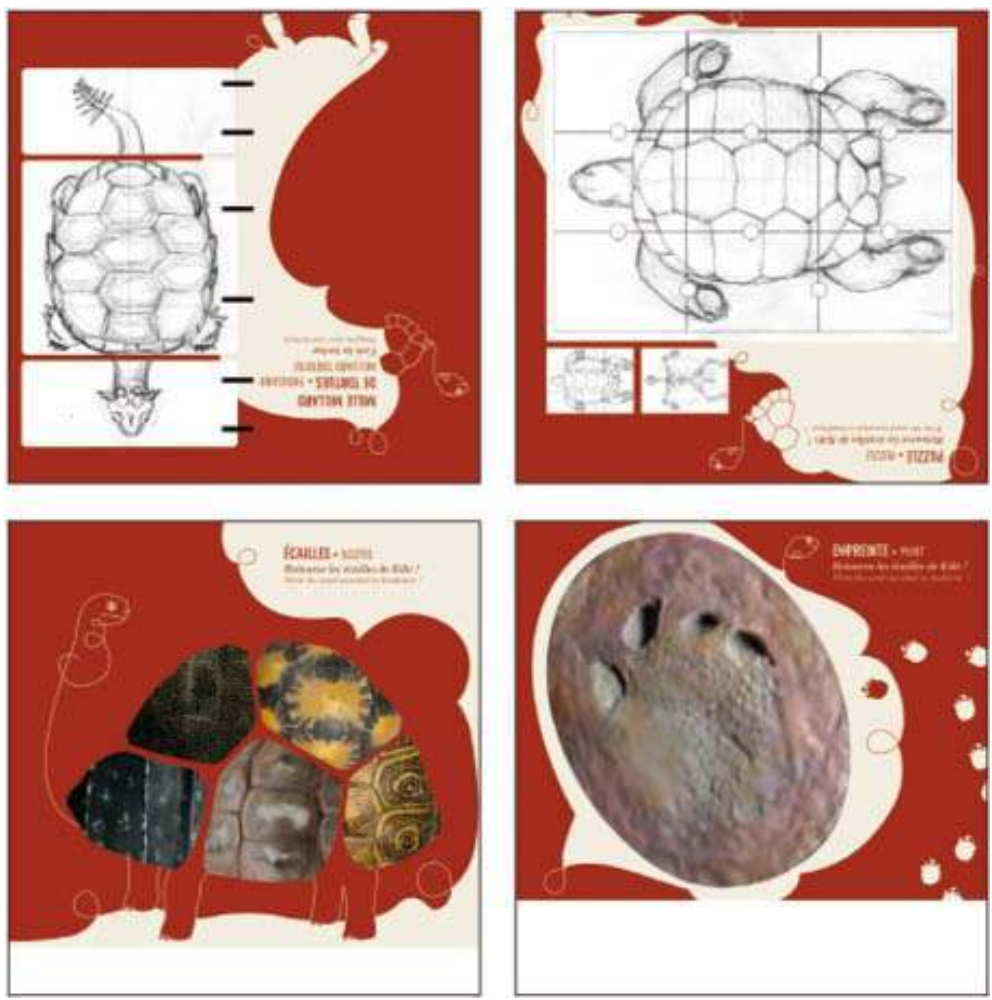

(c) MNHN

Ces trois espaces ont une identité marquée jeune public, mais restent indépendants, chacun ayant une place spécifique dans le parcours général de la GGE. Ces choix s'accompagnent de nombreuses questions dont celle importante de réussir l'intégration de ces espaces dans un parcours général qui se déroule sur plusieurs niveaux.

\section{Les partis pris muséographiques et de médiation}

\section{La cohérence du discours et les débats autour des contenus}

L'intégration de ces espaces dans la trame narrative de l'exposition permanente et du discours qui les accompagne est primordiale.

Par exemple, dans le scénario prévu autour de l'aménagement de l'espace Narval, la question s'est posée d'aborder ou non la chasse des narvals par les Inuits, celle-ci étant une activité traditionnelle qui perdure encore aujourd'hui en des occasions particulières. Il semblait donc très intéressant de l'évoquer, même en l'absence de lien évident avec le parcours permanent. En effet, les milieux marins et polaires de la Grande Galerie présentent la diversité des espèces et des milieux, alors que les relations Homme/Nature ne sont traitées qu'à l'étage supérieur, dans l'espace "L'Homme facteur d'évolution". De même, comment peut-on insérer la nouvelle présentation du narval dans la trame existante des milieux marins et polaires de la Grande Galerie?

Le choix s'est finalement porté sur la valorisation du thème de la chasse du narval par les Inuits à travers un mobilier jeu conçu pour les enfants, autour du langage des Inuits et 
des outils utilisés pour la chasse. Il s'explique avant tout par le fait que l'espace Narval s'intégrait alors au parcours biodiversité centré sur les relations Homme/Nature. Ce parcours n'existant plus aujourd'hui, la question du lien avec la trame générale de la GGE se pose à nouveau.

Des questions du même ordre ont accompagné l'intégration de l'espace consacré à la tortue Kiki, au deuxième étage de la GGE qui présente "L'Homme facteur d'évolution". Le lien narratif existait avec la salle des espèces menacées ou disparues et supposait que le public accède à l'espace Kiki par cette salle.

\section{L'harmonisation graphique et scénographique}

Des interrogations liées à la charte graphique se sont également posées : devait-on se situer en décalage complet avec le reste de la Galerie ? Et quelle cohérence apporter aux trois espaces : le Dodo, le Narval et Kiki?

Le projet autour du dodo étant le premier réalisé, il a donné le ton pour le traitement graphique de l'ensemble. Sa charte graphique qui consiste en une couleur dominante et la silhouette de l'animal "en liseré blanc", a ainsi été conservée.

L'aménagement autour du narval est réalisé avec un éclairage d'ambiance qui suggère l'immersion du narval. Ce type d'éclairage rappelle l'éclairage d'ambiance des milieux terrestres de la GGE imaginée par ses concepteurs et qui fait aujourd'hui l'objet d'un nouveau projet autour de l'évènement des 20 ans de la Grande Galerie.

19 Des photographies ont été intégrées dans les panneaux texte. Elles permettent de contextualiser l'animal dans son milieu, que ce soit le Narval ou la tortue Kiki. Ce choix s'est effectué en marge des souhaits des architectes concepteurs de la GGE qui préconisaient que la mise en scène des milieux marins et terrestres soit conçue de manière suggestive par les lumières, les regroupements des spécimens et dont il ressort une représentation très épurée, sans panneau texte ni photographie. 
L'espace Narval.

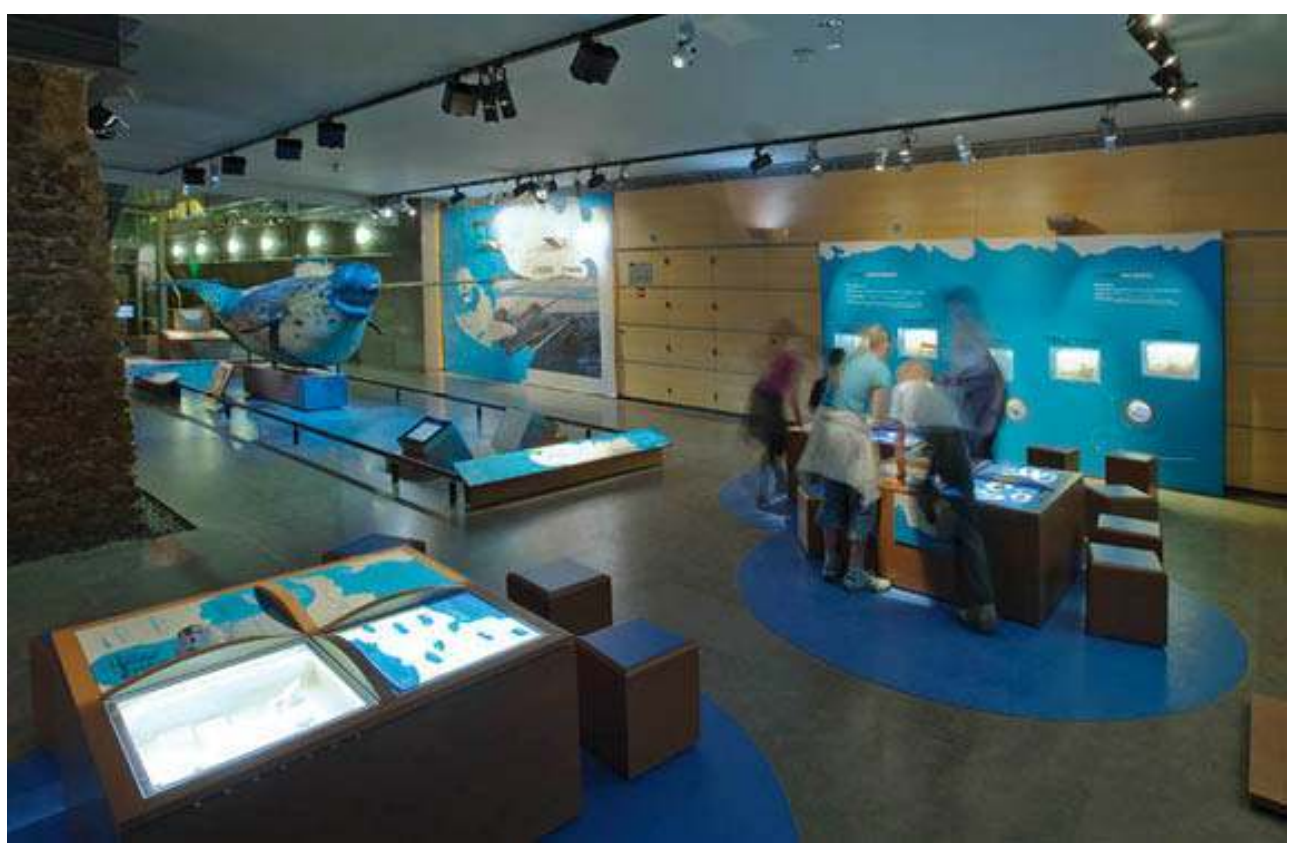

(C) MNHN / L. Bessol et B. Faye

Ces appels graphiques ont néanmoins été déterminants dans la mesure où ils proposent d'emblée une vision différente des graphismes de la GGE. Ils introduisent pour les jeunes publics des images parfois ludiques, voire spectaculaires, permettant une relation différente avec les spécimens présentés. Par exemple, l'idée d'aborder la longue vie de la tortue Kiki sous la forme d'une chronologie requiert une intervention graphique, qui se doit d'être tout de suite perçue par les enfants à l'aide d'images, d'illustrations et de mots clefs. En outre, raconter la vie de Kiki à la ménagerie nécessite l'apport de nombreux documents (photographies au fils du temps, interviews de soigneurs, images de l'évolution de son espace...). À partir de ces différents supports d'information, on peut envisager une multitude de dispositifs, mais les images photographiques d'époque, fort nombreuses, peuvent à elles seules raconter cette histoire. Ce type d'approche, qui n'existait pas jusqu'alors dans la GGE, offre à ces jeunes publics un regard nouveau sur les spécimens présentés.

21 Tout en étant une contrainte, ces espaces isolés du parcours général ont constitué un terrain d'expérimentations pour les choix à venir. En effet, le narval est en lui-même un spécimen isolé, il s'agit d'un plâtre historique de taille imposante. Il devrait être en lien avec la diversité des milieux polaires arctiques mais il se situe en retrait des milieux polaires antarctiques. Les raisons d'un tel choix se comprennent peut-être au regard de sa taille imposante ou par le fait qu'il constitue un spécimen historique que les concepteurs souhaitaient absolument présenter.

De la même manière, l'espace réservé à l'accueil de la tortue géante des Seychelles n'était plus ouvert au public depuis de nombreuses années.

Cet isolement du reste de la GGE a offert une liberté qui a permis d'intégrer des éléments innovants et porteurs de sens pour les enfants. 


\section{Le public des enfants} "mobilier jeu" pour les enfants. s'inscrivent dans cette logique.

\section{Une évaluation de l'aménagement de l'espace Narval}

Des dispositifs ont aussi été conçus spécifiquement pour les enfants, par exemple le théâtre animé de l'espace Dodo qui illustre l'histoire de la dissémination de l'animal. Le petit cabinet de curiosité de l'espace Kiki ou encore l'ajout dans l'espace Narval d'un igloo

Enfin, les mobiliers "fiches de lecture" de la GGE ont été copiés et transformés en

Une première évaluation de l'espace Narval a été menée en 2010 par l'observatoire des publics du Muséum national d'Histoire naturelle. Elle visait à mesurer la perception de cet espace par les publics auxquels il est d'abord destiné : les adultes accompagnés d'enfants. La méthodologie s'est appuyée sur l'observation des comportements et des parcours des visiteurs afin de déterminer l'attractivité de cet espace et des différents éléments qui le composent. 33 groupes de visiteurs - enfants avec adultes - ont ainsi été suivis à leur insu dans l'espace en question. Le temps passé, le parcours emprunté et les points d'arrêt ont été notés, de même que leur attitude vis-à-vis des supports et objets muséographiques.

L'étude révèle que le temps de visite du lieu par les adultes est estimé à 10 minutes ; celui des enfants s'élève à 16 minutes. Les principaux résultats relatifs à l'attractivité du lieu font apparaître que, sur 10 visiteurs, 2 explorent l'ensemble de l'espace de présentation du narval, 5 la partie de droite seulement tandis que les 3 autres se limitent à la zone d'entrée située devant le narval. Il apparait également que la zone de gauche "licorne de terre, licorne de mer" est très peu fréquentée, avec seulement 2 visiteurs sur 10. Pour y remédier, l'igloo a été introduit dans le but d'augmenter l'attractivité autour de cette partie. Une évaluation complémentaire pourrait préciser l'effet bénéfique ou non de cette remédiation.

L'étude a également permis de mettre en évidence différents types de parents accompagnateurs. Les passifs, qui représentent 1 visiteur sur 10, se contentent de suivre ou d'attendre les enfants sans lire ou intervenir auprès d'eux. Certains visiteurs (1 sur 10) lisent et s'informent pour eux-mêmes, laissant les enfants se débrouiller seuls. Les pressés (4 sur 10) accompagnent les enfants tout en les incitant à poursuivre la visite. Enfin les incitatifs et coopératifs ( 4 sur 10), aident les enfants à répondre, lisent les textes avec eux, les interpellent. 
Le plan de l'organisation thématique et spatiale de l'espace Narval.

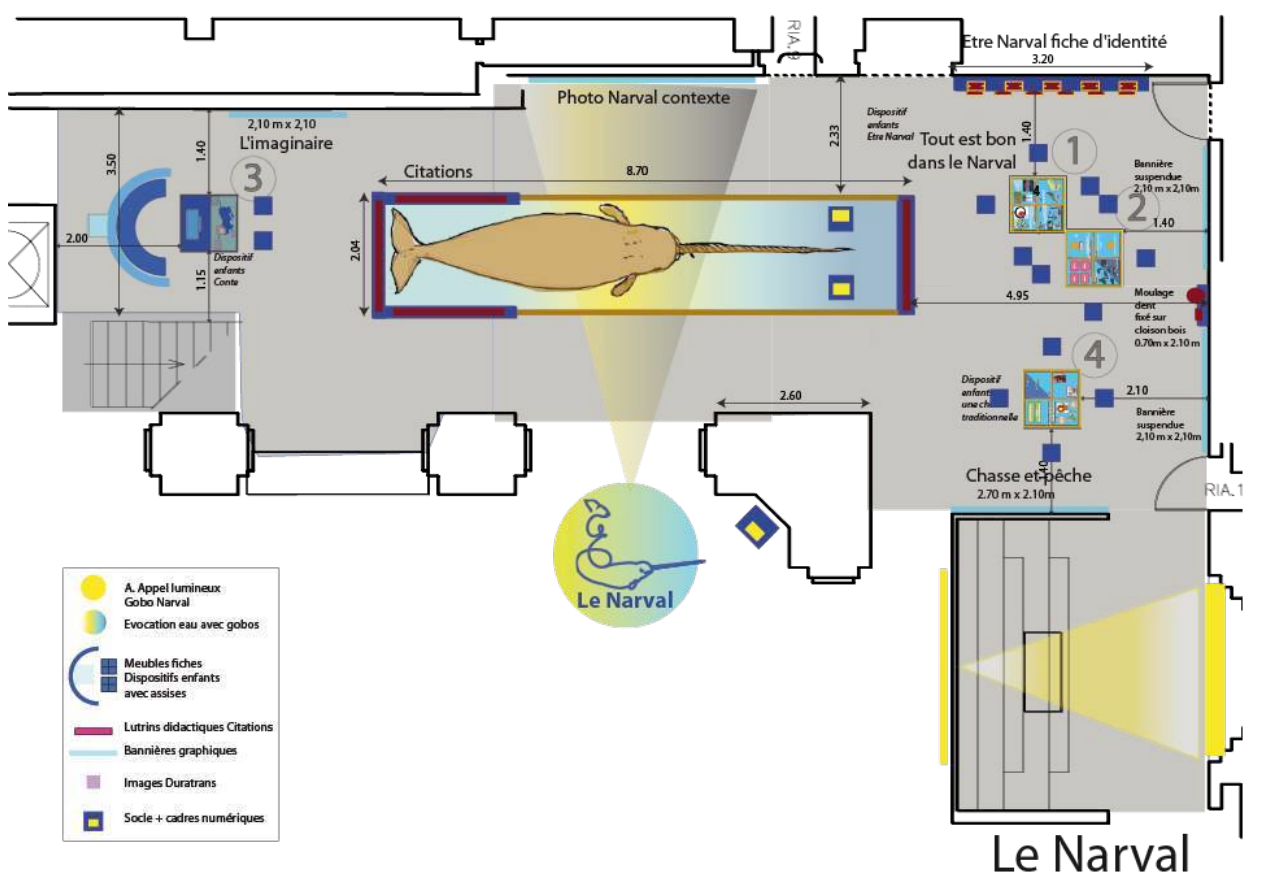

(c) MNHN 
Presque 20 ans après l'ouverture de la GGE, il serait sans doute souhaitable que l'exposition permanente évolue plus globalement, mais "il est certes plus facile de théoriser sur ce que doivent être les expositions dans nos établissements que de démontrer dans la pratique ce qui doit être fait pour qu'elles soient effectivement continues et évolutives" (D. Jammot, L. Péru, 1987).

lors, que devient le sens de ces espaces au regard des conditions de production initiales?

Si l'on réfléchit à leur place actuelle dans la structuration spatiale de l'immense Grande Galerie, ces trois espaces sont éloignés les uns des autres. L'espace Narval est situé au rezde-chaussée de la GGE alors que les deux autres espaces se situent au deuxième étage. L'un apparaît donc en début du parcours de visite tandis que les deux autres espaces se situent à sa fin, à un étage où les visiteurs accompagnés de jeunes enfants ne vont pas forcément. En effet, après avoir traversé les deux premiers niveaux de la GGE qui traitent de la biodiversité marine et terrestre, bien souvent le temps de visite supportable pour les jeunes enfants est écoulé. Ces deux espaces deviennent donc des espaces autonomes qui peuvent être découverts et visités indépendamment, et dont l'identité est moins celle d'un parcours à proprement parler que de petits espaces qui jalonnent des parcours de visite possibles, petits espaces dont l'usage doit être accompagné, prescrit ou conseillé par les équipes de la GGE.

C'est l'intérêt et la place de propositions pour les enfants dans la GGE qui donne aujourd'hui leur vie, leur intérêt et leur devenir à ces espaces, pour l'équipe de la GGE comme pour les visiteurs. La réflexion sur la place de ces espaces dans des parcours doit se poursuivre et intégrer d'autres productions, notamment la médiation et l'information, pour redécouper la structuration spatiale de la GGE en types de visite possibles, spécialement adaptées pour les enfants.

Afin de faciliter l'identification de ces espaces, ils ont été intégrés dans le plan de la GGE distribué aux visiteurs. La plaquette quadrimestrielle de l'établissement fait également référence à l'espace Kiki, en mentionnant son adaptation pour le jeune public et les familles. Enfin le nouvel album de la Grande Galerie intègre ces espaces dans les pages "visiter la Grande Galerie avec des enfants".

D'autres pistes sont envisagées, comme la présentation de l'ensemble des points "spécifiques enfants" via un document de type flyer ou d'une affiche à l'accueil. Ils donneront une information sur l'existence de ces lieux et la possibilité de les intégrer dans le parcours de visite de la GGE.

3 En outre, la médiation humaine au sein de ces espaces a été renforcée. Une visite atelier "Le narval, quel animal étrange" est ainsi proposée aux classes maternelles. Accompagnée par un médiateur, elle permet une exploitation de l'espace et un lien avec les milieux polaires puisque le temps de découverte de l'espace Narval est lié à une visite introductive des milieux polaires de la GGE.

39 La conception d'un parcours muséographique évolue désormais vers des propositions nouvelles d'accompagnement des pratiques de visite avec enfants ou en famille, propositions qui s'appuient sur ce que font les visiteurs de ces espaces et sur l'effort de recréation de modalités de visite dans l'existant. 


\section{BIBLIOGRAPHIE}

Jammot, D. et Péru, L. Problématique des expositions permanentes dans les musées de sciences naturelles de province, Muséum, vol. 154, n² 2, 1987, pp. 102-106.

Lafon, F. Restitution des suivis de parcours de groupes de visiteurs adultes et enfants dans

l'espace Narval - Parcours biodiversité - GGE. Muséum national d'Histoire naturelle, Direction de la diffusion, de la communication, de l'accueil et des partenariats, septembre 2010.

\section{RÉSUMÉS}

Après avoir rappelé quelques étapes de la création d'espaces d'interprétation pour le jeune public au Muséum national d'Histoire naturelle, l'auteur met en évidence - à travers les choix muséographiques effectués et les types de médiations proposés - les difficultés d'intégration de ces espaces au parcours de l'exposition permanente et les questions relatives à un accompagnement porteur de sens pour les enfants.

\section{INDEX}

Mots-clés : parcours, enfant

\section{AUTEUR}

\section{FABIENNE NOÉ-STOSIC}

chargée de projets jeune public, responsable de l'action pédagogique et culturelle au Département des Galeries du Muséum national d'Histoire naturelle.

noe@mnhn.fr 